\title{
La sexualité des Hindous anciens et l'apport de Sir Richard Burton (1821-1890) à son étude
}

\author{
Georges ANDROUTSOS, Aristide DIAMANTIS, Dimitrios PLOUMPIDIS
}

Histoire de la Médecine, Faculté de Médecine, Université d'Athènes, Athènes, Grèce

\begin{abstract}
RÉSUMÉ
Cet article traite des mœurs sexuelles des anciens Hindous par rapport aux religions dominantes à chaque époque. En outre, sont présentées et analysées les trois œuvres principales de l'érotologie Hindoue et la contribution de Richard Burton à leur mise au jour.
\end{abstract}

Mots clés : sexualité des anciens Hindous, Kama Soutra, Richard Burton

\section{RELIGIONS HINDOUES ET SEXUALITE}

L'hindouisme, ou brahmanisme, arriva en Inde avec les Aryens du Caucase. La mythologie hindoue a proliféré sur place en un assez exténuant foisonnement, avec des sectes toujours vivaces. Etablissant des castes infranchissables, conférant un pouvoir moral incontournable aux brahmanes, l'hindouisme continue de dominer la vie des Hindous.

Pour l'hindouisme, le sexe est presque sacré car il est essentiel à la vie et digne par conséquent d'être étudié sérieusement. Les plaisirs, selon Vatsyayana, l'auteur du Kama Soutra, « sont nécessaires pour le bien-être du corps autant que la nourriture; ils sont donc tous les deux également indispensables ". Une grande différence avec la tradition judéo-chrétienne qui associe le péché et le sexe d'une façon obsessionnelle [14].

Le bouddhisme inaugure la conception virginale. La mère du Bouddha Maya vivait dans la chasteté conjugale. Un éléphant la féconda en lui perçant le flanc droit d'un coup de défense : l'enfant Gauthama (Bouddha) sortira aussi par le flanc, laissant à la mère intact son précieux hymen et sa virginité. Bouddha s'élève, vers 600 , contre la dictature des brahmanes. Il prêche une doctrine à la fois populiste et élitiste, unissant le mouvement cosmique et la recherche du Nirvana immuable grâce à la méditation, fondant un ordre monastique où règne le mépris des choses terrestres [7].

Le jaïnisme, héritier des croyances pré-aryennes, est un athéisme plus qu'une religion. II croit à la transmigration des âmes, dont le douloureux cheminement de corps en corps peut être stoppé par le sacrifice, l'ascèse, la chasteté, la non-violence et le végétarisme : il ne faut surtout pas supprimer la moindre vie animale [6]...

Quant aux vertus roboratives du régime végétarien, elles sont nulles en ce qui concerne la puissance sexuelle; bien au contraire la carence en protéines animales prive l'organisme d'aliments énergétiques normaux pour fabriquer des hormones, nourrir les muscles et le système nerveux central. Le légume n'est pas le remède de l'impuissance.

Bien que rivaux, hindouisme et bouddhisme se retrouvent dans leur commune misogynie. Et dans le paradoxe qui leur a fait élaborer des techniques permettant, par l'acte sexuel lui-même, de se libérer de la femme et du désir qu'elle inspire.

L'hindouisme reconnaît dans le désir, kama, le moteur de l'univers. Désir sexuel, engendrant le plaisir d'amour, la joie, etc. Cet amour est célébré dans les hymnes de l'Atharva Véda, guide de la famille heureuse. Famille dont l'homme est le pivot, et où l'infidélité n'est pas tolérée. Les adultères avaient le nez tranché, ce qui a fait développer l'opération de reconstitution du nez par mobilisation d'un lambeau cutané frontal (Figure 1).

Le brahmanisme méprise les femmes. II les considère comme perverses, paresseuses, ignorantes. Selon les lois de Manou, les orifices féminins sont purs au-dessus de la ceinture, impurs au-dessous. Malgré toute l'utilité reconnue à la yoni, le sacré lingam est l'organe sexuel le plus vénéré en Inde.

Correspondance :

Pr Georges ANDROUTSOS - 1 rue Ipeirou, 10433, Athènes, Grèce. Email lyon 48@otenet.gr 


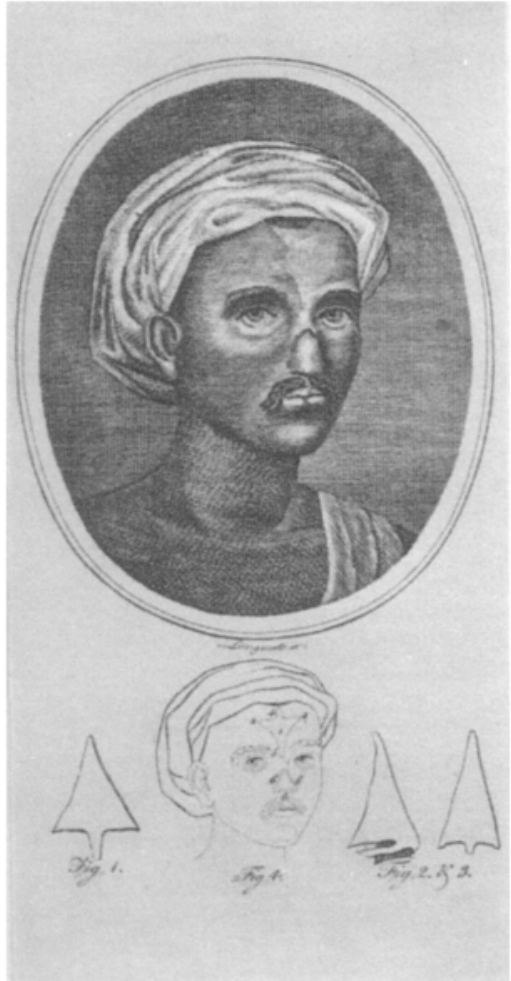

Figure 1 : Le procédé hindou de reconstitution d'un nez.

C'est la secte shivaïste qui introduisit avec ses prescriptions ou tantras, de l'érotique délibéré dans la religion. Le tantrisme est en partie issu d'une antique religion locale vouée à une très classique Grande Déesse. Cette Sakti va devenir l'épouse du dieu Shiva. Comme toute Grande Déesse qui se respecte, elle a des aspects peu amènes. Dourga ou Kali exigeant des sacrifices humains, en particulier de petites filles. Et finalement elle incarne le principe femelle origine de tous les maux de l'homme.

Certes le tantrisme voit la dualité mâle-femelle jusque dans le corps humain, Shiva logeant dans la tête (le lotus céphalique) et Sakti dans la région sacro-coccygienne (le lotus du cul). Chacun tient sous sa dépendance un des côtés du corps, avec un « vaisseau " particulier de part et d'autre de l'échine (moelle), à droite le " bon " vaisseau masculin à gauche le vilain vaisseau féminin. L'union sexuelle permet de faire passer le souffle des deux vaisseaux latéraux dans le vaisseau central pour engendrer la félicité. En traitant le mal par le mal.

La femme et le vin sont les poisons de l'homme. En prescrivant de boire (modérément) et de s'accoupler, Shiva se conduit en classique homéopathe. Le poison vainc le poison !

De bons croyants ont pu ainsi organiser de petites fêtes sexuelles entre amis. Le couple des hôtes s'installait au centre du cercle formé par les couples des invités. On mangeait, buvait et copulait très " religieusement ».

II fallut mille ans pour que s'apaisent les frictions entre la contestation bouddhiste et l'hindouisme traditionnel des brahmanes. L'infiltration de l'un par l'autre aboutit, en matière de sexualité, à ce très étonnant vajrayana - le véhicule de foudre - qui mélange copulation, fumigation de cervelle et exploitation de la femme.

Pour le bouddhisme, le corps est le véhicule obligatoire conduisant à la vérité. Vérité qui procure la félicité suprême, dans la méditation extatique coupée de tout mouvement. L'accouplement prolongé, sans émission externe de sperme, est le moyen privilégié pour engendrer le processus de méditation. Bel éloge de l'incarnation humaine.

Le tantrisme, inspiré du tao chinois [2], l'unit à la doctrine hindouiste des deux courants nerveux véhiculant l'énergie le long de la moelle épinière : le courant femelle et le courant mâle. Tant qu'ils restent séparés l'individu (mâle) ne peut parvenir à l'extase. Il faut les réunir par la formation du bindou. Cet esprit volatil se forme pendant l'accouplement, grâce au mélange de l'énergie masculine avec l'énergie féminine. Le bon tantrique sait conserver son érection des heures durant. II capte l'énergie femelle dans le vagin de sa partenaire aux ordres et grâce à sa technique de pranayama, qui permet de retenir son souffle, il retient l'émission externe du sperme. Montant vers les hautes sphères après la réunification des deux canaux spinaux sous l'intercession du bindou, l'énergie virile de la semence rebrousse chemin. Elle parvient au sommet de la tête où elle éclate dans le lotus céphalique. Sa splendeur provoque alors l'" identification » du pratiquant avec le vide, et aussi avec la divinité : c'est le Nirvana! autrement dit le coitus reservatus.

Ici divergent deux écoles. Les tantristes façon tao tiennent à la présence réelle de la femme. L'important demeure qu'elle soit munie de sa yoni ! [11].

L'autre école est purement autochtone, et s'inspire de la vénérable pratique du hata yoga. On sait que cette discipline enseigne très sérieusement 168 postures pour s'accoupler. De plus, les yogis savent depuis longtemps retenir leur souffle, donc leur semence. Ils savent aussi aspirer par l'urètre l'eau des récipients témoins. Ils pourraient donc véritablement aspirer les humeurs vaginales - l'énergie féminine. Mais point ne leur est besoin de cet appoint externe. Le yogi sait réveiller l'énergie femelle qu'il porte en lui-même, la koundalini, le serpent lové sous son coccyx (dans le lotus du cul). Un bon moyen d'animer l'animal dormant consiste à jouer de la flûte pour faire dresser la tête d'un cobra. Energie femelle et énergie mâle se réunissent alors pour emprunter un nouveau réseau nerveux asexué, où s'engage la semence non répandue. Elle remonte vers le turban, et l'illumination embrase le cerveau.

On se demande comment un peuple qui paraît posséder une telle somme de prescriptions copulatoires pourrait souffrir de pannes. La crainte de l'échec se manifeste pourtant de façon très ancienne, dès l'Arthava Véda, qui comporte une hymne pour favoriser la puissance virile. Et point n'est besoin de rappeler que les Indes furent l'antique et célèbre terre de culture des aphrodisiaques. Les consommateurs locaux de piment et de curry entendaient bien se garantir contre la mollesse du lingam. Et c'est finalement à cette rubrique de la prévention que toute la gymnastique spiritualo-fornicatrice peut posséder quelque utilité. Puisque la médecine locale « 
traditionnelle " s'est toujours effacée devant les brahmanes [10].

La thérapeutique sexologique ne peut retenir des techniques du yoga que les pratiques s'apparentant à ce que l'Occident appelle « relaxation ", pour lesquelles il a élaboré lui aussi des méthodes efficaces et documentées.

Cela décrispe les inquiets et peut atténuer les troubles purement fonctionnels. On sait aussi que l'exercice régulier est le meilleur moyen pour conserver la forme érotique.

En fait, en dehors de la classe aisée sachant lire, possédant maison, pièces closes, temps libre, on imagine mal les millions et les millions d'hindous démunis, pauvres paysans, parias mendiants, exécuter consciencieusement leurs exercices de Kama Soutra. Bien au contraire les perpétuelles grossesses, les perpétuels allaitements rendent les porteuses de yoni indisponibles pendant des mois et des mois.

Nées à l'époque de la domination absolue des aristocrates, toutes les théories contorsionnistes et rétentionnistes n'ont jamais été pratiquées que par les princes et leur entourage. Plus à titre de passe-temps que d'édification morale [14].

\section{CRÉMATION DES VEUVES : UNE COUTUME HINDOUE BARBARE}

La coutume sexuelle la plus atroce vient du pays de la résignation, de l'Inde. La sati [3], la crémation des veuves, n'est que l'union de l'épouse à son mari poussée dans ses dernières conséquences. La femme unie à un homme doit rester éternellement avec lui. L'affliction, le culte du mort, la renonciation à un remariage ne suffisent pas. Une femme vraiment attachée à son époux ne peut se séparer de lui corporellement. S'il est appelé par la mort dans un autre monde, elle doit suivre le même chemin et mêler ses cendres aux siennes, le jour même où le mari est incinéré. Cette conception a des accents lyriques et héroïques à la fois : elle professe que le mystère du mariage, que la fidélité conjugale sont plus fortes que la crainte de la mort. Mais le geste perd un peu de sa générosité si l'on réfléchit qu'il est la conséquence de la servitude sexuelle inouie dans laquelle l'homme et la société ont jeté la femme. II fait une impression encore plus fâcheuse si l'on connaît les circonstances dans lesquelles cette coutume s'est développée et s'est conservée jusqu'à une époque récente.

La crémation des veuves remonte au moins au lle millénaire av. J.-C. Elle n'a jamais été répandue dans l'Inde toute entière. Elle vient des tribus de langue aryenne ; la population dravidienne du Sud ne s'en est jamais vraiment accommodée. On est tenté de rattacher cette coutume aux sacrifices humains religieux, mais les plus anciens livres sacrés de l'Inde, les Védas s'en écartent déjà. Ils la tolèrent tout au plus et recommandent vivement de sauver les femmes de la mort par le feu. Si une femme se couche sur le bûcher à côté du cadavre de son époux et qu'à la dernière minute un autre homme lui saisisse la main, elle doit le reconnaître pour son second mari et retourner au monde des vivants. D'ailleurs cet excellent conseil ne semble pas avoir été souvent suivi ; il fallait que l'homme s'échappât aussi vite que la femme et donc que tous deux eussent prémédité ce singulier mariage sur un bûcher.
Si les prescriptions religieuses ne sont pas responsables de la crémation des veuves, les prêtres portent une lourde responsabilité. Les brahmanes exhortaient surtout les femmes riches à se faire brûler avec leur mari pour récolter leur l'héritage. L'autodafé des femmes pauvres, où ils n'avaient rien à gagner, n'était pas nécessaire. Le prêtre ne se souciait pas de celles qui vivaient en polyandrie. L'incinération des veuves restait un privilège des castes supérieures. Quand régnait chez elles la polygynie, il arrivait que plusieurs femmes se fissent brûler simultanément lors du décès de leur seigneur et maître, dans l'attente de renaître un jour avec lui. De tels cas s'observaient encore au $19 \mathrm{e}$ siècle, même après que le vice-roi britannique, lord William Bentinck, eût interdit en 1829 l'incinération des veuves.

Au milieu de ces pratiques absurdes, associées à des captations d'héritages, un fait apparait déjà comme un trait de lumière : on limita du moins la Sati aux adultes. Dans les castes supérieures il était de bon ton que l'on cherchât pour des fillettes en bas âge un époux de même âge. Les filles étaient considérées dans l'Inde, à toute époque, comme une charge peu enviable pour les parents : plus tôt on s'en débarrassait, mieux cela valait. Si un mari de 3 ans mourait (rougeole), sa promise était considérée comme veuve et devait le rester toute sa vie.

Certes, au cours de l'histoire de l'Inde, la position de la femme n'est pas toujours restée la même. Il y eut pour elle, notamment sous l'influence bouddhiste, des périodes de liberté relative, et d'autres d'oppression plus dure. Sous la dynastie Goupta, aux IVe et Ve siècles, époque de civilisation raffinée, il y eut des femmes employées dans des postes administratifs élevés. Mais cela n'exclut pas qu'au même moment la polygynie se répandit, que le célibat des veuves fût observé avec hostilité et que leur crémation fût pratiquée couramment [5].

\section{KAMA SOUTRA : L'ACTUALITÉ D'UN LIVRE SUR L'AMOUR QUI A PLUS DE 2000 ANS}

Le Kama Soutra fut écrit au VIle siècle par Vatsyayana Mallanaga. Vatsyayana n'était pas un libertin frivole, mais passait pour un homme sage et pieux, et la précision pédante avec laquelle il initie son lecteur aux secrets de l'érotique, justifie parfaitement cette réputation. La légende conte que ce fut à l'instigation de son prince, pour le divertissement de la cour. Tout le monde a lu ses passages réputés érotiques. Ses descriptions alambiquées de baisers plus farfelus les uns que les autres. Son classement des hommes et des femmes selon la dimension de leurs organes génitaux. Ses catalogues de postures copulatoires. En fait, tout ce chapitre copulatoire n'est qu'un traité de bilboquet, énumérant mille et une façons d'enfiler le lingam dans la yoni [12].

Que les femmes aient parfois besoin de jouir autrement que par l'enfilage rituel est envisagé, quand même, par un autre traité, l'Ananga-Ranga [4]. Le clitoris y est nommé. Comme il figure, vilain et conique, dans la vulve ou, encore plus vilaine, triangulaire à pointe inférieure.

Kama Soutra signifie littéralement : " Préceptes d'amour», et Vatsyayana est un instructeur qui va au fond des choses. Dans sa présentation, ce manuel d'érotique ne se distingue 
pas fondamentalement des traités que l'Inde ancienne nous a laissés sur l'art de la guerre, l'art de gouverner, l'art dramatique, etc. L'acte sexuel, avec tous ses préliminaires, est aussi un art, que l'on doit étudier scientifiquement pour atteindre le maximum de jouissance. C'est exactement le contraire du Nirvana bouddhiste : I'homme doit vivre pour les joies de ses sens et les savourer toutes [13].

Remarquons d'ailleurs que l'art de l'érotique n'est pas seulement destiné à préparer le plaisir de l'homme. II doit aussi procurer à la femme le summum de la volupté. Vatsyayana prétend même que la femme peut parvenir à des joies plus intenses que l'homme, car se donner est pour elle la volupté de prendre conscience de sa valeur personnelle. Mais son rôle n'est nullement passif, elle s'anime par les stimulations de toute sorte de son partenaire, afin d'atteindre elle-même la pleine réalisation de ses désirs.

Les Hindous aiment les nombres, les dénombrements minutieux en toute chose. Des nombres d'ailleurs allègent et remplacent même souvent la description. Aussi dans le Kama Soutra, tous les processus imaginables de l'amour, psychologiques aussi bien que physiologiques, sont résumés en schémas numériques. Il y a trois types fondamentaux de couples amoureux, que Vatsyayana caractérise par des comparaisons zoologiques : lièvre et gazelle, taureau et cavale, étalon et éléphante. Pour faire sa cour, l'homme peut entrer en contact physique avec la femme de quatre manières différentes. Quand les relations amoureuses ont déjà fait quelque progrès, on en vient alors à une série graduée de dix baisers, qui vont du baiser symbolique de l'image dans l'eau au plus raffiné baiser colombin. La technique des étreintes amoureuses est traitée avec autant de détails. La haute école de l'érotique Hindoue en distingue quatre et leur donne des noms poétiques : enlacement de lianes, ascension de l'arbre, embrassement du sésame et du riz et embrassement du lait et de l'eau. Par la combinaison des baisers et des embrassements, on arrive à 64 possibilités pour les jeux préliminaires. Pour l'acte sexuel lui-même, on donne des indications de détail de toute sorte et de nombreuses variantes. Cependant, au bout du compte, on peut bien dire que la technique indienne de l'amour ne dépasse pas ce qu'en d'autres pays les jeunes couples d'amants découvrent sans étude scientifique préalable [9].

Vatsyayana avait lui-même l'impression de n'avoir pas fait encore assez pour ses lecteurs avec ses préceptes à l'usage de tous. II y ajouta donc un "Enseignement secret ", consacré notamment aux médicaments pour renforcer la puissance virile, pour vaincre la frigidité féminine, pour égaliser les tempéraments ou encore pour préserver les femmes de l'infidélité. La pharmacie de ménage du Kama Soutra est très riche; elle va de drogues employées encore aujourd'hui comme aphrodisiaques jusqu'à des excréments de singe.

Comme l'usage en a persisté depuis dans ce genre de littérature, Vatsyayana ne s'enveloppe pas seulement d'un manteau scientifique, mais d'un voile de moralité. Homme pieux, il recommande expressément d'implorer toujours la bénédiction des brahmanes dans l'acte sexuel, mais de ne jamais s'y livrer dans leur voisinage, ni dans un temple de l'amour. Car, à la différence des religions de l'Asie antérieure, l'hindouisme ignore la prostitution dans les temples. Les prêtres Hindous ont à cet égard pour règle de ne pas mêler les affaires à la vie sexuelle [5].

La conception de Vatsyayana, franche et libérée de toute culpabilité, décrit ainsi sa femme idéale : "Ses yeux sont brillants et beaux comme ceux d'une biche. Ses seins sont durs, pleins et dressés, son yoni ressemble à une fleur de lotus qui s'entrouvre et la semence de son amour a l'odeur du lys à peine éclos, elle marche comme un cygne et sa voix émet une musique suave...». Cette conception, comparée à celle exprimée par Saint Jean dans son Apocalypse, met en évidence le fait que Jean n'aimait pas les femmes alors que Vatsyayana les appréciait.

Le Kama Soutra est l'œuvre la plus célèbre qui ait été écrite sur le sexe. Bien que seule la deuxième partie de la version de Sir Richard Burton traite exclusivement de sexe, telle est l'idée que l'on se fait du Kama Soutra dans l'imaginaire collectif. Mais en réalité, le Kama Soutra recèle de nombreux autres thèmes.

Le contenu sexuel des trois œuvres maîtresses de l'art d'aimer indienne représente le lien le plus direct entre ces cultures et la nôtre. Le sexe représente un excellent moyen pour commencer à comprendre une autre culture, comme il l'est pour commencer à comprendre une autre personne. Plus simplement : existe-t-il quelque chose de plus intéressant que de lire comment se faisait l'amour en Inde au moment de la naissance des Evangiles (Kama Soutra), ou au moment où Christophe Colomb découvrait le Nouveau Monde (AnangaRanga), ou encore ce qui déclenchait et stimulait l'érotisme du Grand Vizir de Tunis alors que de l'autre côté de la Méditerranée le pape Borgia se livrait à des jeux illicites avec sa fille Lucrèce (Le Jardin Parfumé) ?

Sir Richard Burton et ses amis de la Kama Shastra Society publièrent le Kama Soutra en 1883. Mais pendant plus d'un demi-siècle un cercle limité de savants et de bibliophiles connut l'existence de ce classique. C'est seulement dans les années soixante du $20^{\ominus}$ siècle, que le Kama Soutra connut une vaste diffusion.

Malheureusement, le livre rejoignit la longue liste des classiques non lus. En général, le Kama Soutra est considéré comme un manuel sur le sexe ou, pire encore, comme un livre pornographique. Rien ne pourrait être moins pornographique que cette œuvre, qui ne peut cependant être considérée comme un livre où est enseigné l'amour pas à pas. En effet, de nombreuses positions pour faire l'amour ne sont praticables que par des yogis experts ou du moins par des athlètes exceptionnellement souples. Vouloir faire l'amour en suivant des recettes tout comme dans un livre de cuisine, en s'en remettant surtout aux jeux préliminaires, a eu pour conséquence de fixer, dans l'imagination des hommes, l'idée erronée d'un clitoris, jusque là ignoré, qui fonctionnerait comme une sorte de manette de démarrage ; or ceci est complètement étranger à l'esprit du Kama Soutra. 


\section{ETUDE COMPARATIVE DES TROIS CEUVRES PRINCIPALES DE LA LITTÉRATURE ÉROTIQUE HINDOUE}

Le Kama Soutra est la plus importante et la plus ancienne des trois ceuvres majeures de l'art d'aimer indienne. Les sources de l'auteur Vatsyayana sont le vaste patrimoine de l'érotologie hindoue du 1er siècle apr. J.-C.

Le soutra est un aphorisme. Le soutra était très employé certainement du fait de la diffusion restreinte de l'écriture et permettait aux étudiants de mémoriser plus facilement des textes. Toutes les œuvres importantes sont rédigées en sanscrit et sont écrites sous forme de soutras.

Grand connaisseur du monde sans en être dégoûté, Vatsyayana traite l'argument avec un détachement parfois presque clinique. Cependant, son livre est avant tout un ouvrage humain, un chef d'œuvre de tolérance et de bon sens. Malgré « l'opposition de certains sages "Vatsyayana insiste pour que les femmes lisent ce livre et sur ce thème disserte comme d'habitude avec pragmatisme et humanité.

Tous les auteurs Hindous qui lui succédèrent ne purent que suivre ses traces ; d'ailleurs, au cours des siècles, les moins importants d'entre eux le reconnaissent : avec le Kama Soutra, Vatsyayana a écrit une des plus grandes œuvres de la littérature mondiale.

L'Ananga-Ranga, ou Condition de Celui qui est Incorporel, est une œuvre du Moyen Age indien. Que de changements étaient intervenus depuis l'époque de Vatsyayana ! Dans l'Inde antique la ségrégation des femmes n'existait pas et les rapports sexuels avant le mariage et extraconjugaux étaient de toute évidence habituels. Kalyana Malla, l'auteur de l'Ananga-Ranga, vivait dans une société rigide et gouvernée par des règles précises, dans laquelle le mariage en bas âge était la norme ; les hommes et les femmes qui n'étaient liés d'aucune manière, avaient peu de possibilités de s'approcher.

La société était soumise à de nombreuses règles de comportement et l'on retrouve la même situation dans l'Ananga-Ranga. Les préceptes sont d'une minutie impressionnante dans les détails : "Pendant la nouvelle lune, le yoni de la femme doit être palpé jusqu'à ce qu'elle s'entrouvre comme une fleur ". Des instructions aussi détaillées indiquent comment traiter chaque partie du corps de chaque type de femme durant les huit parties de la journée et de la nuit pendant tout le calendrier lunaire. II ne fallait surtout pas commettre d'erreur !

Les interdictions sont tout aussi impressionnantes : Kalyana Malla énumère une série d'interdictions concernant ce qui ne peut pas se faire, les lieux où l'on ne doit pas le faire et les personnes avec lesquelles il ne faut surtout pas penser pouvoir le faire, sur un ton qui parfois frise l'hystérie.

Tout en étant pédant et en ayant une bonne opinion de luimême, Kalyana Malla poursuit le louable objectif d'empêcher que les lois rigides du mariage de son temps ne rendent celui-ci opprimant pour les deux époux. A signaler que certains conseils sur le sexe sont meilleurs que ceux de Vatsyayana.

Les deux hommes ne destinaient pas leurs écrits au même public. Vatsyayana voulait inspirer les rapports entre les amants alors que Kalyana Malla s'adressait à des hommes mariés. Même si l'Ananga-Ranga est inférieur au Kama Soutra, son intention explicite de démontrer qu'un unique partenaire sexuel est suffisant pour tout un chacun le rend plus actuel dans la société d'aujourd'hui menacée par le virus du SIDA.

Les trois livres érotiques ont tous été écrits par des hommes. II est incontestable que chaque auteur était en quelque sorte un produit de la société dans laquelle il vivait en tant qu'homme, mais ce sont justement ces différences d'attitude et de contenu qui rendent encore plus heureux le rapprochement de ces trois manuels opéré dans l'édition publiée par la Kama Shastra Society. Mais, alors que Vatsyayana et Kalyana Malla étaient des hommes qui écrivaient pour un public d'hommes et de femmes, le cheikh Nefzawi auteur du Jardin Parfumé [8] était un homme qui écrivait pour des hommes à la manière d'un vieux vicieux. Mais il était plus poète que les deux autres auteurs réunis et avait de l'humour, qualité presque toujours absente dans la plupart des textes érotiques.

Malgré les 14 siècles qui séparent l'Ananga-Ranga du Kama Soutra, ils appartiennent tous les deux à la même ligne culturelle : les similitudes entre les deux mondes ne sont pas moins surprenantes que les différences. La capacité de l'Inde d'assimiler la culture des différents envahisseurs est immense : chaque nouvelle influence a été peu à peu absorbée pour devenir partie intégrante de l'Inde. Le Jardin Parfumé provient en revanche d'une culture différente. Tunis, où le cheikh Nefzawi écrivit le Jardin Parfumé, se démenait en effet dans un précaire équilibre entre les cultures espagnole, musulmane et ottomane. Dans cette ville riche et fastueuse, le cheikh Nefzawi a trouvé les conditions idéales pour composer son œuvre.

\section{SIR RICHARD BURTON : SA VIE ET SON APPORT À LA MISE AU JOUR DE L'ÉROTOLOGIE HINDOUE}

Un nombre incroyable d'écrivains a tenté de décrire la vie et l'œuvre de Burton. Aucun n'a réussi à le faire !

L'aspect physique de Richard Burton (Figure 2) donne une idée de la manière dont il impressionnait les gens. Il existe de nombreux portraits de Burton, tous éloquents, mais les descriptions de ceux qui l'ont connu sont elles aussi très intéressantes. Le voyageur et poète Wilfrid Blunt écrivit : « sa façon de s'habiller et son aspect tenaient plus du forçat remis en liberté que d'une personne de bonne réputation. II me faisait parfois penser à une panthère noire, prisonnière dans une cage mais implacable ; d'autres fois, avec son crâne rasé et son physique de fer, il me rappelait cette formidable créature de Balzac, l'ex-forçat Vautrin qui cachait son identité louche sous les habits d'un abbé. En général il endossait un par-dessus noir délavé avec une écharpe en soie noire toute froissée ; il ne portait pas de col : avec sa musculature puissante et son thorax démesuré, cet accoutrement le rendait singulier et particulièrement laid ; de plus son visage mat, cruel, méfiant avec des yeux semblables à ceux d'une bête féroce, produisait une impression sinistre que je n'avais jamais vue ». Le critique 


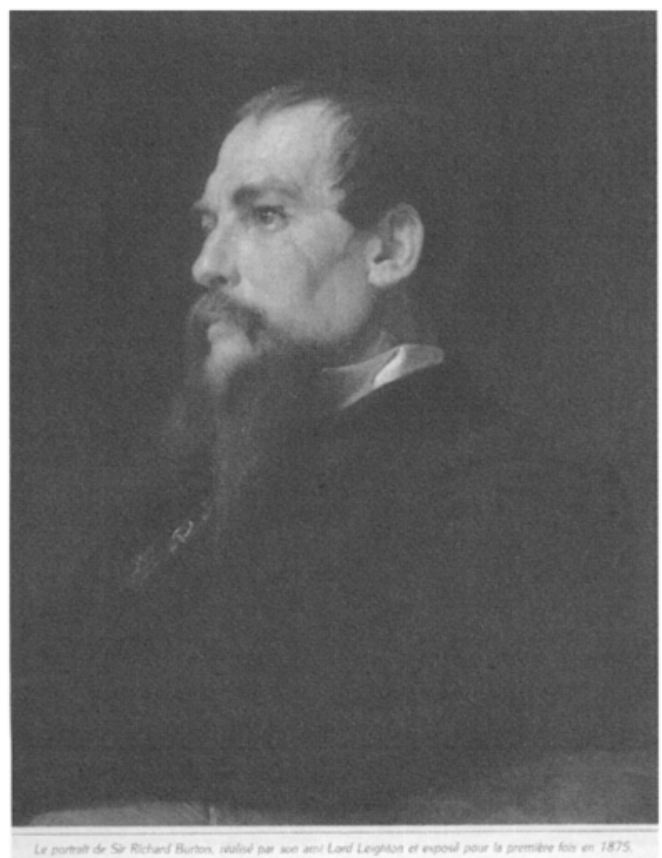

Figure 2 : Sir Richard Burton.

Arthur Symons parle d'une « bouche tourmentée par le désir, avec des narines dilatées qui aspiraient je ne sais quels étranges parfums ".

Cette description de Blunt et de Symons se réfère à Burton à quarante ans, quand son expérience du monde et quelques cicatrices lui avaient donné un certain charme satanique.

Expulsé d'Oxford pour s'être battu en duel et avoir refusé de se conformer au règlement, Burton s'enrôla dans l'Infanterie indigène de Bombay en 1842 à Baroda. Détesté par ses collègues officiers, il eut plus de succès auprès d'une fille hindoue qui partageait son bungalow et il fut surnommé " le nègre blanc $"$.

Ce ne fut pas son style de vie privée mais sa passion pour la culture et les langues orientales qui lui valurent ce surnom. Cependant sa tendance à s'habiller comme un indigène et son penchant pour les langues indiennes qu'il maniait avec facilité pour passer inaperçu de nuit dans le « bazar " ont eux aussi contribué à l'existence de ce surnom. Ces capacités donnèrent l'idée au général Sir Charles Napier d'utiliser Burton comme espion. Ce rôle s'adaptait parfaitement tant à la personnalité qu'aux dons de ce jeune officier : secret et dangereux, il lui faisait toucher de près les pulsions intimes de la vie ; lui seul était capable de faire de telles choses. Mais, comme cela arrivait souvent avec Burton, l'éclat qui aveugla les autres finit par se retourner contre lui. II entreprit beaucoup de choses qui suffiraient à remplir une douzaine de vies, mais souvent, quand tout semblait répondre à ses attentes, il rétrogradait au point de départ à cause des démons qui faisaient partie de sa personnalité.

A Karachi, Burton fut chargé par Napier d'enquêter sur les maisons de prostitution car la syphilis menaçait l'efficacité des militaires et Napier voulait avoir une idée claire sur la situation. II obtint un résultat qui allait bien au-delà de ce à quoi il s'attendait ! Le général, fameux pour sa dureté, fut profondément surpris en apprenant que trois bordels étaient organisés pour une clientèle homosexuelle. Sa naïveté dut surprendre Burton qui fut néanmoins chargé de rédiger un rapport exhaustif et réservé afin de faire fermer ces lieux.

Burton rédigea aussi un rapport riche de détails sur les pratiques des eunuques, des jeunes gens et sur les requêtes des clients.

L'armée n'a jamais été le lieu qui correspondait à Burton. Trois ans supplémentaires en Inde furent trop et Burton, déprimé et malade, repartit pour l'Angleterre en 1849.

Durant la décennie qui suivit, Burton finit deux des plus grandes entreprises de sa vie, celles pour lesquelles il devint célèbre. En 1853, déguisé en pèlerin, il se rendit à La Mecque. Ce n'était pas le premier des infidèles à avoir le courage de courir ce terrible risque, mais le récit de ces expériences constitue une œuvre merveilleuse.

Puis commença la période de l'exploration de l'Afrique qui culmina avec le plus célèbre de ses voyages : l'expédition entreprise avec John Speke à la recherche de la source du Nil. Après avoir affronté des épreuves et des dangers terribles, Speke et Burton découvrirent le lac Tanganyika. Cette espèce de vaste mer, à l'intérieur du continent, devait sûrement être, selon eux, la source du Nil et, après une rapide reconnaissance, ils revinrent à la ville de Kazeh. Là ils se reposèrent et se préparèrent au voyage de retour vers la côte. Speke cependant voulait continuer l'exploration vers le Nord avant de repartir : le bruit circulait qu'il existait un autre lac. Burton refusa de l'accompagner et Speke dut y aller seul. Le destin se répéta : alors qu'il tenait dans ses mains le succès total, Burton le laissa échapper. En effet ce fut Speke, et non Burton, qui découvrit le lac Victoria, la vraie source du Nil.

Ce ne sera pas la dernière fois qu'une telle situation se répètera. Des années plus tard, alors que l'amertume et la jalousie suscitées par l'expédition du Nil, et la mystérieuse mort de Speke n'étaient plus que de lointains souvenir et, après avoir accompli une série de missions sur ordre du « Foreign Office " qui n'étaient pas adaptées à ses capacités, il fut nommé consul à Damas. II s'approchait alors de la cinquantaine, ses conditions de santé n'étant pas des meilleures, cela pouvait être le dernier poste idéal pour un passionné d'études arabes. Ne tenant pas compte des merveilleuses possibilités que cet endroit recelait pour lui, il fut muté à Trieste où il mourra dix-huit ans plus tard.

Isabelle soutenait inlassablement son mari qui devenait toujours plus irascible et intraitable. A Trieste, il étudia des questions en rapport avec le monde entier. Dans une grande salle étaient disposées plusieurs tables ; sur chacune d'entre elles s'accumulait le matériel relatif à chaque livre, de telle manière que Burton pouvait passer de l'une à l'autre selon I'humeur du moment, comme il l'avait fait dans le temps, se déplaçant d'un continent à l'autre.

S'ils croyaient l'avoir freiné, il leur aurait vite démontré qu'ils avaient tort et que c'était une folie de penser que ses expéditions étaient terminées. D'ailleurs, il y avait des lieux cachés de la littérature hindoue, et lui seul possédait la clé 
pour pénétrer dans le jardin parfumé...

La porte du bureau de Burton était très souvent close pour Isabelle. Cette femme dévote et conventionnelle ne s'intéressait pas au fait de savoir si les Arabes appellent l'anus « grenade » ou si l'infibulation est pratiquée par telle ou telle tribu. En revanche, de telles connaissances exotiques intéressaient particulièrement certains savants : les membres de la Kama Shastra Society.

La Kama Shastra Society n'était pas moins mystérieuse que son fondateur. Les fausses pistes et les culs de sac dans lesquels elle s'engageait parfois n'étaient pas seulement dus à l'amour du mystère si cher à Burton, mais à une protection nécessaire pour entreprendre la publication de littérature érotique.

A la fin de l'ère victorienne, les acheteurs de livres formaient un vaste public dont une partie considérable était intéressée par le matériel exotique. Des maisons d'édition publiaient des livres pour des lecteurs de tous niveaux, depuis le pire type de pornographie jusqu'à la littérature érotique et aux œuvres sérieuses écrites par des savants.

Le dimanche 19 octobre 1890, en rentrant de la messe, Isabelle trouva Burton qui travaillait à la dernière page du Jardin Parfumé. Elle ne savait rien à ce sujet, mais il s'agissait d'une nouvelle traduction d'une section complète omise dans la version originale de la Kama Shastra Society qui parlait d'homosexualité et de pédérastie. L'état de santé de Burton déclinait depuis quelque temps : un médecin habitait en permanence avec eux dans leur maison de Trieste. Deux jours plus tôt, il avait raconté à Isabelle qu'un oiseau avait tapé du bec sur la fenêtre et il avait fait ce commentaire : " $\mathrm{Ce}$ petit oiseau est de mauvais augure ". Elle avait refusé d'y croire et lui avait fait remarquer que les oiseaux avaient l'habitude de venir prendre de la nourriture sur le bord de la fenêtre.

Mystérieusement, Burton répliqua : « Oui, mais pas à cette fenêtre, à une autre " .

Son destin s'accomplissait, il sentait qu'il allait mourir. II a dû ressentir un grand plaisir quand il s'aperçut que cette fois-ci le succès ne lui échapperait pas au dernier moment et qu'il n'avait pas été leurré à la fin de sa vie. Il avait terminé son travail sur la nouvelle et très importante section du Jardin Parfumé. Les retouches finales du travail furent apportées précisément ce dimanche-là. Burton mourut avant l'aube du lendemain.

Quand Isabelle lut le manuscrit, elle fut prise de panique, craignant pour la réputation de son mari. Elle jeta au feu le manuscrit, plus de mille pages [1].

\section{EPILOGUE}

L'absence de tout sentiment de culpabilité et de péché à l'égard du sexe est peut-être le message le plus important que le lecteur occidental moderne peut recevoir du Kama Soutra et des autres chefs d'œuvres de l'érotologie hindoue.

Les névroses, le malheur ou un comportement morbide par rapport au sexe ne sont pas les seuls maux transmis par ceux qui voudraient nous faire croire que péché et sexe sont synonymes. L'impulsion de la sexualité est trop forte : ceux qui désirent la dominer, en réalité la condamnent. Le Marquis de Sade a écrit : " Ils m'ont convaincu que seul le vice peut faire éprouver à l'homme ces vibrations morales et physiques qui sont la source de la plus délicieuse des voluptés ».

\section{RÉFÉRENCES}

1. BURTON R. Sir : Introduction. In : Le Kama Soutra illustré. AnangaRanga. Le Jardin parfumé. Rome, Gremese International, 1993 : 10-16.

2. CHANG J. : Le Tao de l'art d'aimer. Paris, Calmann-Lévy, 1977.

3. FRAZER R.W. : Sati (article). In : Encyclopaedia of Religion and Ethics. London, James Hastings, 1920, V, XI : 207.

4. KALYANA MALLA : L'Ananga-Ranga. In : Le Kama Soutra illustré. Ananga-Ranga. Le Jardin parfumé. Rome, Gremese International, $1993: 67-112$.

5. LEWINSOHN R. : Histoire de la vie sexuelle. Paris, Payot, 1957 : $37-40$.

6. MAJUMBAR R.C., RAYCHANDHURI H.C., KALIKINKAR DATTA : An advanced History of India, $1^{\text {re }}$ partie : Ancient India. London, Mac Millan, 1949 : 197.

7. MASSON-OURSEL H., DE WILMANN-GRABOVSKA H., STERN P. : L'Inde antique et la civilisation indienne. Paris, La Renaissance du Livre, $1933: 84$.

8. NEFZAWI (Cheikh) : Le Jardin Parfumé. In : Le Kama Soutra illustré. Ananga-Ranga. Le Jardin parfumé. Rome, Gremese International, 1993: 113-156.

9. THOMPSON E. : Sutee. Boston, Houghton Mittlin Co, 1928 : 32-35.

10. THORWALD J. : Histoire de la médecine dans l'antiquité. Paris, Hachette, $1966 ; 178-220$.

11. VAN GULIK R. : La vie sexuelle dans la Chine ancienne. Paris, NRF, $1971:$ 152-154.

12. VATSYAYANA : Le Kama Soutra, Paris, Editeur Tchou, 1980.

13. VATSYAYANA : Le Kama Soutra. In : Le Kama Soutra illustré. Ananga-Ranga. Le Jardin parfumé. Rome, Gremese International, $1993 ; 17-66$.

14. ZWANG G. : Histoires des peines de sexe. Paris, Maloine, 1994 ; 57-62.

Manuscrit reçu : Juin 2008 ; accepté juin 2008. 


\section{ABSTRACT}

Sexuality of the Ancient Indians and the contribution of Sir Richard Burton (1821-1890)

Georges ANDROUTSOS, Aristide DIAMANTIS, Dimitrios PLOUMPIDIS

This article describes the sexual practices of the Ancient Indians in relation to the dominant religions over the ages.

The three major works of Indian erotology and the contribution of Sir Richard Burton to their elucidation are also discussed.

Key words: sexuality of the ancient Indians, Kama Sutra, Richard Burton 